\title{
Perceived Synchronization of Olfactory Multimedia
}

\author{
Gheorghita Ghinea, Member, IEEE and Oluwakemi A. Ademoye
}

\begin{abstract}
The concept of synchronization is of fundamental importance in multimedia systems and applications. The focus of this work reported in this paper is on olfactory enhanced multimedia, which concerns itself with associating computer generated smell with other media. However, the lingering nature of smell, as opposed to the transitory nature of other media objects that multimedia applications are accustomed to, such as video and audio, means that specific attention needs to be given when synchronizing other media content with olfactory data. Consequently, this paper presents the results of an experimental study carried out to explore and investigate the temporal boundaries within which olfactory data output in an olfactionenhanced multimedia application can be successfully synchronized with other media objects from an end user perspective. Results show the presence of two main synchronization regions, and that olfaction ahead of audiovisual content is more tolerable than olfaction behind content.
\end{abstract}

\section{INTRODUCTION}

$\mathrm{I}^{\mathrm{N}}$ $\mathrm{N}$ general, multimedia systems and applications may include any combination of traditional media objects such as text and graphical images, as well as non-traditional media such as audio and video. Synchronization is concerned with defining and maintaining the temporal relationships between two or more correlated media objects that are combined, processed and presented together to produce a multimedia system or application. Because multimedia is essentially about using these multiple media objects to communicate information to users via the component media objects as well as by the relationship between the synchronized media objects [2], [14], [15], [17]-[18], achieving synchronization between them is vital to the success of these systems.

Traditional media objects such as text and still images are time-independent as they are static in nature, unlike audio and video which are time-dependent media objects and are presented as a media stream. However, because synchronization defines temporal relations, at least one timedependent media object should be present when specifying multimedia synchronization. For example, a slide show made up of slides and audio commentary involves synchronization between time-independent (the slides) and time-dependent (audio) media objects, while the synchronization of audio with video in everyday TV, videos or DVDs involves synchronization between time-dependent media objects [2]. Likewise, synchronization may be required between the component units within a single time-dependent media object, e.g. between the frames that make up a video or samples of an audio stream. As such, it is common to distinguish between intra-media object synchronization (the temporal relations between the component units within a single time-dependent media object) and inter-media object synchronization (synchronization between different media objects that need to be presented together simultaneously or in sequence).

While synchronization has been traditionally associated with Quality of Service (QoS) parameters such as Delay, Delay Jitter and Inter-media Skew, in recent times, there has however been a paradigm shift in the multimedia research field with the recognition that technical parameters alone are not sufficient measures to develop and manage algorithms to produce the highest user-perceived quality experience in multimedia applications. Such algorithms must also incorporate measures of the user-perceived quality experience [7][10][22].

From a synchronization viewpoint, the foremost study carried out in this respect was the one undertaken by Steinmetz [2], [17]-[18]. He investigated user-perceived synchronization requirements for lip synchronization and pointer synchronization, as different synchronization requirement results had been reported by different research groups. The purpose of these experiments was to discover the boundaries within which inter-media skew between audio and video data, for the case of lip synchronization, and between audio and pointer is tolerable to the user, that is, the user perceives the combined media objects as being synchronized and does not notice the presence of any jitter. It was shown that while people are sensitive to the correct timing of combined media, users are still able to tolerate multimedia presentations that are not perfectly synchronized to a certain degree.

Whilst one does not expect the inter-media synchronization requirements between olfactory data and other media objects to be as stringent as that required by lip synchronization, i.e. between audio and video, smell has its own peculiarities that will indeed impact on its synchronization requirements, and forms the focus of this paper, which is structured as follows: in section II, we introduce the concept of olfactory data synchronization; sections III and IV then present the details of our experimental methodology and the results of our experiment respectively. Lastly, section $\mathrm{V}$ discusses our conclusions and directions for future research

\section{OLFACTORY DATA SYNCHRONIZATION}

Olfactory data is virtual data, unlike other media streams which have the ability to be stored in some computer data form. Smell, moreover, has a tendency to linger, and its emission usually happens at a slow pace, since it relies on atmospheric breeze to move it through the air. As such, smell is again different from the media objects multimedia applications have become accustomed to i.e. audio, visual and traditional media (text, images, animations), whose component media streams have a transitory nature and do not experience this natural delay. In addition, the occurrence of data loss with olfactory data is also something to be expected, as sensitivity to the presence of a smell tends to decrease with prolonged 
exposure to it; moreover, the presence of atmospheric breeze may cause the olfactory data to waft off in the wrong direction and miss the intended target.

Thus, although computer generated smell is time-based, with the ability to explicitly specify its start time and duration, the characteristic nature of smell means that the actual user perception of the occurrence of the start time, $s$, and its duration, $\mathrm{d}$, is more likely to be $\mathrm{s}+\delta_{\mathrm{s}}$ and $\mathrm{d}+\delta_{\mathrm{d}}$, where typically $0 \leq \delta_{\mathrm{s}}$ and $\mathrm{d}+\delta_{\mathrm{d}} \geq 0$, where $-\mathrm{d} \leq \delta_{\mathrm{d}} \leq 0$ or $\delta_{\mathrm{d}} \geq 0$. In the latter, it means that the user perceived duration of the olfactory data output may be less than or greater than the intended duration, equal to the intended duration, or may be non-existent at all. In the case of the user-perceived start time, interestingly it is also possible to have the scenario where $0 \geq$ $\delta_{\mathrm{s}}$ may be true, where this is a factor of the olfactory generating device, or may be attributed to the characteristic nature of smell and the way the human sense of smell works. Thus, the questions that arise are: should one factor in $\delta_{\mathrm{s}}$ and $\delta_{\mathrm{d}}$ when specifying temporal relationships involving olfactory media data? And if yes, what values should be used?

As a result of these difficulties associated with odour perception, as well as the virtual nature of olfactory data, controlling the parametric values used for measuring synchronization for olfactory data will be quite difficult, as most of these values are more dependent on external factors than they are on the multimedia application or computing environment for olfactory data. For example, controlling delay for olfactory data is more dependent on external factors such as the time it takes the output device to emit the required scent or the effects of nature. Also, as mentioned above, olfactory data itself cannot be transmitted via distributed systems and therefore delay jitter is of little or no relevance for olfactory data, as it will only apply to the commands used to trigger its execution. Bit error and packet loss, like delay, are also more dependent on external factors, Mother Nature and the users' sense of smell in this case. However, inter-media skew is something that must be controlled for any media object that needs to be synchronized as achieving the desired temporal relations between the combined media objects is the core purpose of synchronization. As such, inter-media skew is the only measure of real significance that can be used and optimised whilst determining olfactory data synchronization requirements and it is chiefly what we have controlled to measure the user-perceived experience of the synchronization effect of an olfactory-enhanced multimedia application in our experimental study.

In the experimental study reported in this paper, we measure the user-perceived experience of the synchronization effect of an olfactory-enhanced multimedia application. Specifically, we are interested in discovering the boundaries within which an end user finds the synchronization of olfactory data combined with audiovisual media data in olfactory-enhanced multimedia applications acceptable.

\section{EXPERIMENTAL METHODOLOGY}

\section{A. Experimental Participants}

A total of 42 participants, 14 females and 28 males, between the ages of 18 and 40, from a wide variety of backgrounds, i.e. undergraduate and postgraduate students from different universities and departments, and both blue and white collar workers, took part in the experimental study.

\section{B. Experimental Materials}

We designed a multimedia presentation display program, which displays visual and audio media content from video clips synchronized with olfactory data (Table I). For this experiment, we have used the Vortex Active scent dispensing system by Dale Air, a UK-based company well known for providing themed aroma displays. The Vortex Active scent dispensing system (shown in Fig. 1) is a personal computer smell dispensing system which uses miniature fans to propel the emitted smells in the right direction. It connects to the computer via a USB port, and we used the USB fan controller application supplied with the device to control the synchronized release of olfactory data during each video playback.

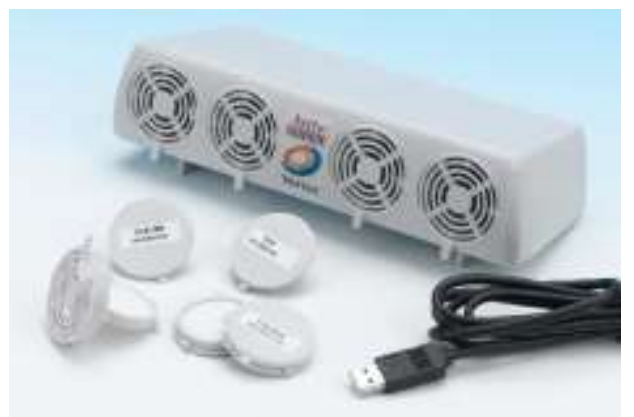

Fig. 1. Vortex Active from http://www.daleair.com/acatalog/Dispensers.html

Despite the distinctive uniqueness associated with smell that makes it possible to detect its presence in the air, research has shown that it is easier for people to smell something than it is for them to identify what they have smelt, and more often than not people will try to identify a smell by associating it with a smell they are familiar with [3]-[4], [11]. Odour identification is also usually influenced by social and cultural factors. For these reasons, and the lack of standard smell classification schemes, it is advised that familiar smells be used for experiments involving the use of olfactory data [11], [16].

We finally opted for six smells widely used in olfactory research [6], [11] as we felt it would not be too difficult to find at least one smell that is easily recognisable and identifiable within each of these 6 smell categories, which incidentally also has a fair distribution ratio between what can be termed as pleasant and unpleasant smell categories. The six smells categories used were flowery, foul, fruity, burnt, resinous and spicy. Table I shows a brief summary of the videos used for each of the smell categories and what smell was generated during its playback. 
TABLE I

VIDEO CATEGORIES AND SMELls USED

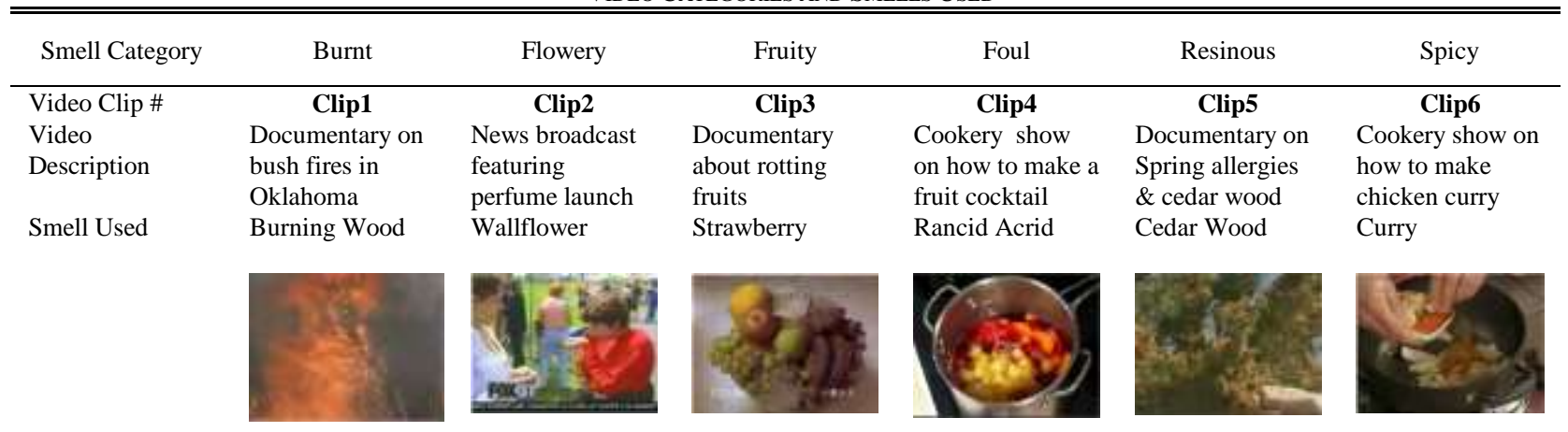

\section{Scented Audiovisual Synchronization Skews}

Scented audiovisual synchronization refers to the temporal relationship between a computer generated stream of smell and audiovisual streams. Perfect synchronization between the olfactory and audiovisual media streams means that the desired temporal relationship is achieved during the olfactionenhanced multimedia presentation. In other words, the olfactory and audiovisual media streams have zero skew between them, as they are in sync. However, as mentioned above, previous research [14], [18] has shown that the presence of inter-media skews between related media streams may still give the illusion and effect of a synchronized presentation. Thus in determining scented audiovisual synchronization requirements, the idea is to discover the acceptable boundaries within which the presentation of the olfactory data in relation to audiovisual content is perceived as being timely by users.

The approach adopted in our study is consistent with the approach carried out by Steinmetz in [18], from which synchronization requirements for lip (audio and video) and pointer (audio and pointer) synchronization were established. Here, artificial skews are introduced between the different media types to be combined in the multimedia application to simulate an out of sync effect and using heuristic methods, the user-perceived quality of experience of the synchronization effect is measured. Measuring the user-perceived experience is a realistic way of establishing synchronization requirements because technically, the presence of even the smallest possible inter-media skew value, say a time shift of a nanosecond between related media, is an out of sync presentation. Thus, there is no way to measure and determine which inter-media skews will give the illusion and effect of an in sync presentation suitable for the end user unless a lot of technical assumptions are made, or as put in [9], "conveniently technical" measures instead of "humanly relevant" measures are used.

Our study was performed by introducing artificially created skews at intervals of 10 seconds ranging from -30 s to +30 s between olfactory stimuli and audiovisual media content. This was achieved by programming our smell dispensing device, the Vortex Active, to start the release of the smell at the skewed start time in relation to its synchronization with the video playback. Steps of 10s were chosen for the following reasons:

- The average time it takes to sniff a scent typically ranges from $0.5 \mathrm{~s}$ to $2.5 \mathrm{~s}$ [12]. However, in cases where when one is expecting an odour to occur as opposed to the natural sudden awareness of odours, the repeated sniffs in anticipation of the odour will typically prolong this.

- Our spectrum range, which we limited to 90 s to accommodate olfactory adaptation. Research [21] has shown that it is best to limit exposure to short varying intervals such as 20 s -30 s rather than long, continuous ones.

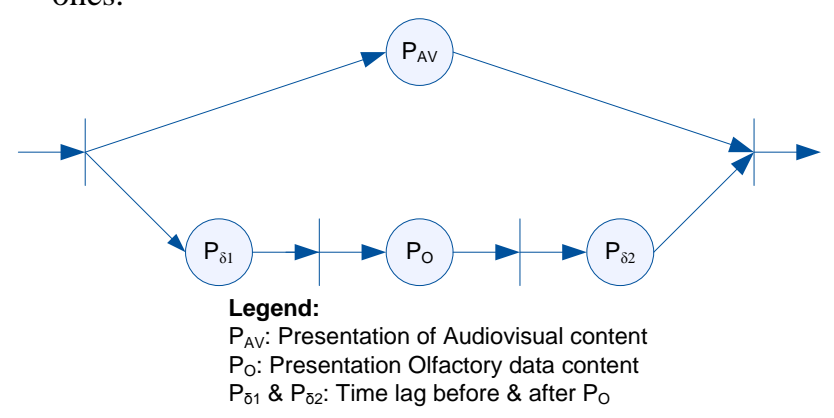

Fig. 2. Temporal Scenario Model for the Olfaction-Enhanced Multimedia Presentations used in the Experiments.

Fig. 2 shows the temporal scenario model for the olfactionenhanced multimedia presentations used in our experiments using the constraint-based Object Composition Petri-Net (OCPN) modelling approach. The time lag before the start of the presentation of the olfactory data in relation to the presentation of the audiovisual content, denoted by $\mathrm{P}_{\delta 1}$ in the figure, is the sum of the desired time lag between the start of $\mathrm{P}_{\mathrm{AV}}$ and $\mathrm{P}_{\mathrm{O}}$ that will provide an in-sync presentation plus the value of the artificially created inter-media skew used in the experimental multimedia presentation.

\section{Experimental Process}

At the beginning of each experiment, participants were told that they were about to view six video clips whose presentation was going to be augmented with olfactory data. Participants were also told that, after watching and experiencing each clip, they would be completing a written questionnaire targeting the 
user experience associated with the respective clip. Once this questionnaire was completed, the next olfaction-enhanced clip would then be played. They were then given the opportunity to address any questions that they might have. Once these (if any) had been addressed, the experiment proper started.

The start viewing times of each video clip was recorded. On average, each experimental case lasted for 1172 s (the fastest time taken to complete the experiment was $733 \mathrm{~s}$, the longest 1404s). Each of the six olfactory enhanced video clips described in Table I was watched in a 240 x 180 window. Each video clip lasted for about 90 seconds, was played and experienced in its entirety, with the VortexActive emitting the corresponding scent for 30 seconds. The excerpts of the video clips were specifically chosen so that audiovisual content relating to the olfactory data the videos were augmented with coincided with the middle $30 \mathrm{~s}$ segment. What differs is whether the olfactory media is released before the middle 30 seconds ('ahead of content', i.e. -30 to -10 seconds skew, namely olfaction released 0,10 , or 20 seconds into the clip), after 30 seconds ('in sync', for which the 30 second aroma coincides with the middle 30 second portion of the clip for which olfactory augmentation is relevant to the content being watched) or after 30 seconds ('behind content', i.e. 10 to 30 seconds skew, namely ofaction released 40,50 or 60 seconds into the clip).

Participants thus viewed each of the olfaction-enhanced video clips with one of the seven defined skew values, including the six artificially introduced skew values and a zero skew value.

TABLE II

INTER-MEDIA SKEW VALUES ASSIGNED TO EXPERIMENT SUBJECT CASES

\begin{tabular}{ccccccc}
\hline \hline Case & $\begin{array}{c}\text { Clip 1 } \\
\text { Skew }\end{array}$ & $\begin{array}{l}\text { Clip 2 } \\
\text { Skew }\end{array}$ & $\begin{array}{l}\text { Clip 3 } \\
\text { Skew }\end{array}$ & $\begin{array}{l}\text { Clip 4 } \\
\text { Skew }\end{array}$ & $\begin{array}{c}\text { Clip 5 } \\
\text { Skew }\end{array}$ & $\begin{array}{l}\text { Clip 6 } \\
\text { Skew }\end{array}$ \\
\hline 1 & $0 \mathrm{~s}$ & $-30 \mathrm{~s}$ & $-20 \mathrm{~s}$ & $-10 \mathrm{~s}$ & $10 \mathrm{~s}$ & $20 \mathrm{~s}$ \\
2 & $30 \mathrm{~s}$ & $0 \mathrm{~s}$ & $-30 \mathrm{~s}$ & $-20 \mathrm{~s}$ & $-10 \mathrm{~s}$ & $10 \mathrm{~s}$ \\
3 & $20 \mathrm{~s}$ & $30 \mathrm{~s}$ & $0 \mathrm{~s}$ & $-30 \mathrm{~s}$ & $-20 \mathrm{~s}$ & $-10 \mathrm{~s}$ \\
4 & $10 \mathrm{~s}$ & $20 \mathrm{~s}$ & $30 \mathrm{~s}$ & $0 \mathrm{~s}$ & $-30 \mathrm{~s}$ & $-20 \mathrm{~s}$ \\
5 & $-10 \mathrm{~s}$ & $10 \mathrm{~s}$ & $20 \mathrm{~s}$ & $30 \mathrm{~s}$ & $0 \mathrm{~s}$ & $-30 \mathrm{~s}$ \\
6 & $-20 \mathrm{~s}$ & $-10 \mathrm{~s}$ & $10 \mathrm{~s}$ & $20 \mathrm{~s}$ & $30 \mathrm{~s}$ & $0 \mathrm{~s}$ \\
7 & $-30 \mathrm{~s}$ & $-20 \mathrm{~s}$ & $-10 \mathrm{~s}$ & $10 \mathrm{~s}$ & $20 \mathrm{~s}$ & $30 \mathrm{~s}$ \\
\hline \hline
\end{tabular}

Subject case 1 applies to participants $1,8,15$, etc., subject case 2 to participants 2, 9, 16, etc. and so on

Table II shows how the introduction of skews in the olfaction-enhanced video clips was split across participants. Thus, each skewed olfaction-enhanced video was watched 6 times. In order to minimize order effects, the playback order of the videos with each of the pre-defined skews was randomized.

\section{E. Physical Layout of Laboratory Experiment Environment}

The experiments were conducted in a usability lab with a door on one side of the room and a large window opposite it (Fig. 3). The experiment was setup in advance of participants arriving for a scheduled experiment, with the door and window being opened whilst this was being done, ensuring that any ambient odours present in the room, which may have interfered with odour perception, were eliminated prior to a participant's arrival.
Each participant was seated along one side of the laboratory, facing the computer used for the display of the multimedia video clips. A keyboard and mouse were provided for navigational purposes. The Vortex Active is placed about half a metre away from the participant, tilted at an angle in line with the participant's nose and mounted on a raised platform. Using this setting it took about 2 s for the scent emitted by the Vortex Active device to reach the target user's nose.

The lab window was left open during the experiment. Moreover, whilst the participant was completing the written questionnaire pertaining to the video clip that had just been watched, the desktop fan was switched on to further eliminate the possibility of smell lingering effects between successive viewings of the six olfaction-enhanced multimedia video excerpts. In pretests we had found that a period of 30s was sufficient, given the open window and the fan, to eliminate all odours (and no participants ever completed the clip questionnaire faster than this). We also used this time interval to, when applicable, load scent cartridges corresponding to the next video clip to be watched (six scents were used in our experiment, but only four cartridges can ever be loaded at any one time in the VortexActive).

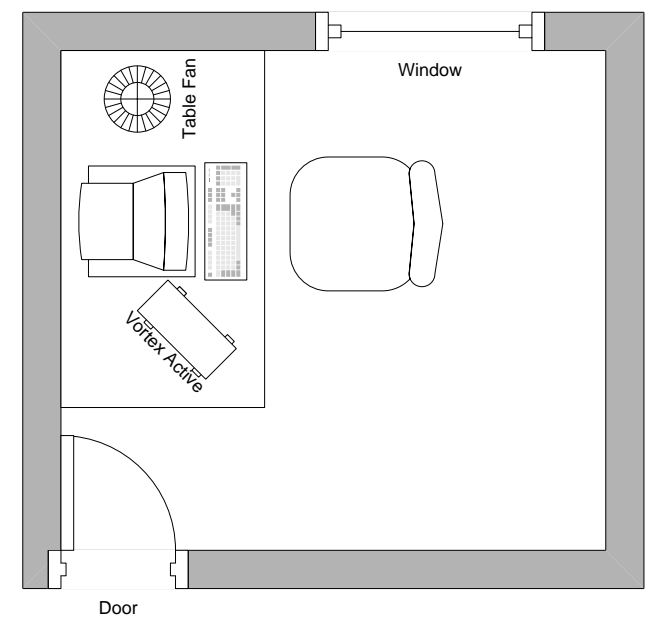

Fig. 3. Graphical Representation of the Physical Layout of the Laboratory Experiment Environment

\section{RESULTS}

\section{A. Acceptable inter-media skew between olfactory and audiovisual media content}

To arrive at answers to the question we set out to answer with our experiment, participants were asked to respond to the statement:

\section{The smell was released}

by choosing one of the options: 'Too Early', 'Early', 'At an Appropriate Time', 'Late' and 'Too Late'.

Analysis of the results using the non-parametric MannWhitney test showed that significant differences in participants' perception of the scented audiovisual synchronisation effect occurred at an inter-media skew of -30 s 
$(\mathrm{z}=-5.105, \mathrm{p}<0.05)$, i.e. olfaction ahead of audiovisual content by 30 s or more, and at an inter-media skew value greater than $+20 \mathrm{~s}(\mathrm{z}=-2.213, \mathrm{p}<0.05$ at $+20 \mathrm{~s}$ and $\mathrm{z}=-2.686, \quad \mathrm{p}<0.05$ at +30 s), i.e. when olfaction is behind audiovisual content by more than 20s. At each of the other artificially introduced inter-media skew values ranging between -20 s to +10 s, the differences in participants' perception of the synchronisation effect were not found to be statistically significant when compared with the case for perfect synchronisation. Consequently, these results reveal that the temporal boundaries for synchronising olfactory media with video media may be split into the following two regions, showing the range in which presence of inter-media skew synchronization errors are acceptable to users:

- The in-sync region: where this region spans between a maximum skew of -30 s, when olfaction is ahead of audiovisual, and a maximum skew of +20 s for when olfaction is behind audiovisual. That is, olfactory media content may be generated up to 30s ahead of audiovisual content or delayed by a maximum of 20s before users begin to notice that there is a synchronisation error present. Accordingly, these temporal boundaries represent the synchronisation requirements when olfactory media content is combined with audiovisual content.

- The out-of-sync region: this region spans beyond a skew of -30 s, when olfaction is ahead of audiovisual content and beyond +20 s when olfaction is behind audiovisual content. Here, the majority of participants detected the synchronization error.

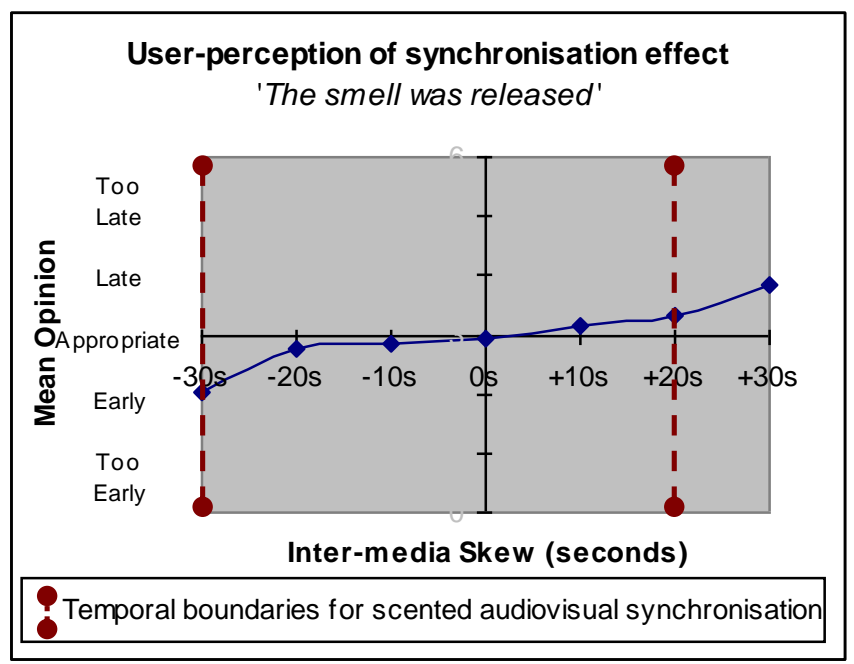

Fig. 4. Overall analysis of synchronisation errors with respect to applied inter-media skew between olfactory data and audio-visual content.

In Fig. 4, we show a graphical representation of these results and also highlight the temporal boundaries for scented audiovisual synchronization requirements established. The left hand side of the graph, indicated by the negative skew values, is for the situation where the olfactory data was ahead of the related audiovisual content and the right hand side represents the situation where the audiovisual content was ahead of the olfactory data. The mean opinions show that although participants noticed the synchronisation error, they generally did not consider the error to be too severe as they were mostly of the opinion that the released smell was released early or late, as opposed to opinions reflecting the more severe impression of being either too early or too late.

Furthermore, the temporal boundaries established show that olfaction behind audiovisual content is more noticeable than the case when olfaction is ahead. This is reflected by the slightly smaller range at which the presence of inter-media skew becomes noticeable when olfaction is behind, i.e. an inter-media skew range of $20 \mathrm{~s}$, compared to an inter-media skew range of 30s when olfaction is ahead. Nonetheless, this is consistent with the nature of smell to sometimes linger and drift when emitted, with the consequence that such a natural delay occurring in the presence of inter-media skew involving olfactory media content will definitely favour the case for when olfaction is ahead of other media content.

\section{B. Impact of Delay on the User-Perceived Experience}

The influence of synchronisation errors on the userperceived experience will be ascertained according to participants' opinions in respect of the following:

- User-perceived experience of the sense of relevance of the olfactory media

- User-perceived experience of the sense of reality of the olfaction-enhanced multimedia

- User acceptability of olfactory media in terms of how distracting or annoying they find it

- User-perceived enjoyment of the olfaction-enhanced multimedia experience

We shall now examine each in turn.

\section{1) Impact of inter-media skew on the perceived sense of} relevance of the olfactory media

To examine the influence of inter-media skew on the perceived sense of relevance of the olfactory media, we analysed participants' agreement with the statement - 'The smell was relevant to what I was watching'. The MannWhitney test was used to compare participants' opinions of the sense of relevance of the olfactory media when a synchronisation error was present with the case for perfect synchronisation showed that there was no significant difference in participants' perception of the sense of relevance of the olfactory media. Moreover, this was also true for skews of -30 s (olfaction ahead) and greater than +20 s (olfaction behind), identified above as the level at which participants' began to notice the synchronisation error (Fig. 5). 


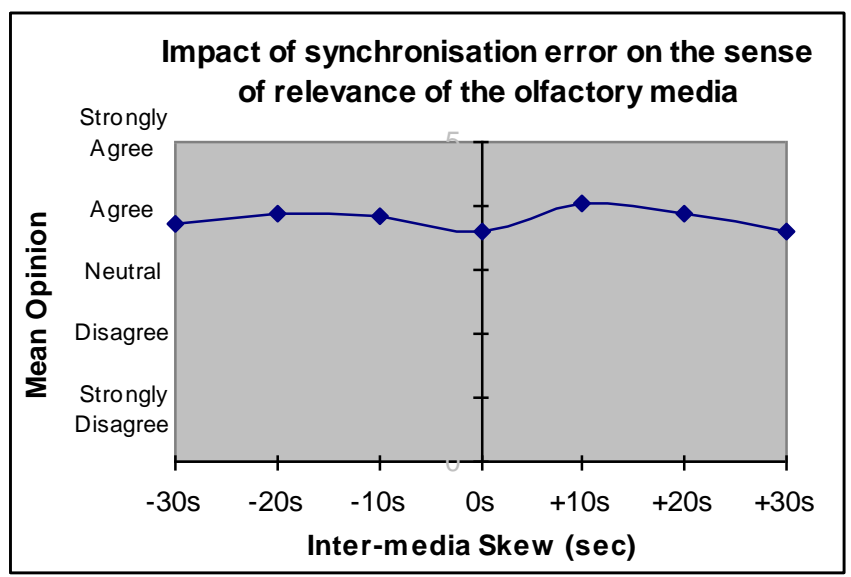

Fig. 5. Impact of inter-media skew on the perceived sense of relevance of the olfactory media

2) Impact of inter-media skew on the perceived sense of reality of the olfactory media

We analysed participants' responses indicating their level of agreement, or disagreement, with the questionnaire statement 'The smell heightened the sense of reality whilst watching the video clip'. Results from Mann-Whitney test showed that with the exception of a skew of $+30 \mathrm{~s}(\mathrm{z}=-2.158, \mathrm{p}<0.05)$, i.e. olfaction $30 \mathrm{~s}$ behind audiovisual content, there was no significant difference in participants' opinions in respect of the sense of reality of the olfaction-enhanced multimedia video clips. Moreover, the mean opinion values, shown in Fig. 6, reflect that generally participants had a positive bias as regards the added olfactory media heightening the sense of reality of the multimedia video excerpts. The curve in Fig. 6, representing the mean opinion values, has a noticeable dip at a skew of +30 s, but otherwise it is relatively straight and also shows that the mean opinions at the different skews introduced are comparable in this respect.

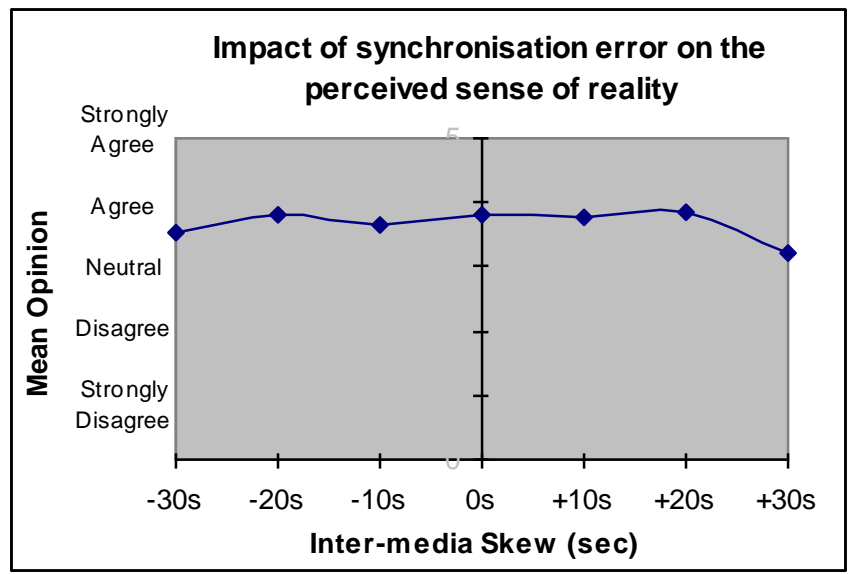

Fig. 6. Impact of inter-media skew on the sense of reality of the olfactionenhanced multimedia video clips

\section{3) Impact of inter-media skew on the acceptability of the olfactory media}

To measure the impact of inter-media skew on the acceptability of olfactory media, we measure how distracting and annoying participants found the presence of out-of-sync olfactory media in the olfaction-enhanced multimedia video clips. In order to do this, we analyse participants' responses to the questionnaire statements, 'The smell was distracting' and 'The smell was annoying' at each of the inter-media skew values introduced for the different video clips.

In this respect, the results obtained from a Mann-Whitney test found the olfactory media used to enhance the video clips revealed that there was no significant different in participants' opinions of how annoying and/or distracting the olfactory media in the presence and absence of inter-media skew. Thus, even the skew values identified as being out-of-sync by participants (as identified above) did not result in a more distracting or annoying experience than the case for perfect synchronisation or at the skews still perceived as being insync. Next we analyse the means obtained in respect of these results.

The mean opinions reflecting how distracting and annoying participants found the olfactory media, both in the presence and absence of inter-media skew, are shown in Fig. 7 and Fig. 8 respectively. Generally, the mean opinion values reflect that participants mostly had a negative bias, or were neutral, in their opinions of how distracting and annoying the olfactory media used to enhance the multimedia video clips was. Moreover, this perception was the same both in the presence and absence of inter-media skew between the olfactory media and video media content.

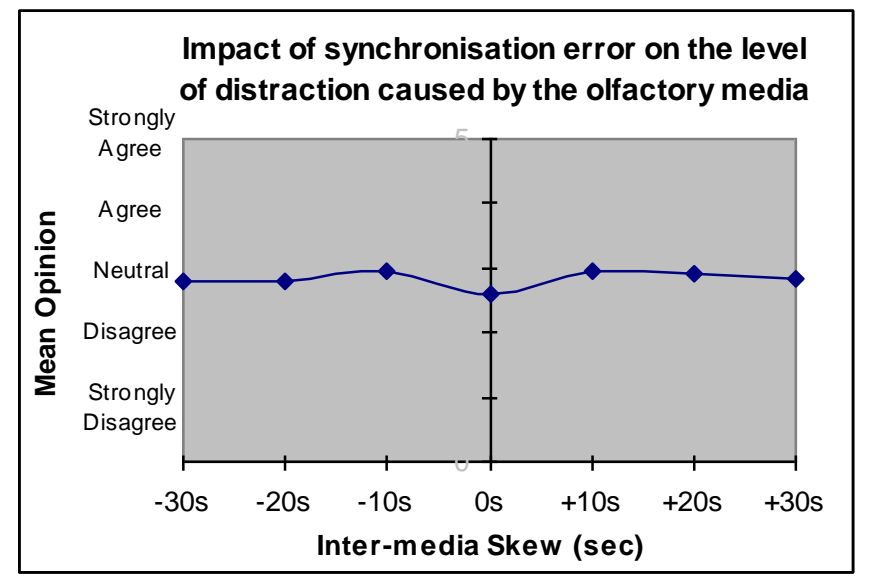

Fig. 7. Impact of inter-media skew on the perceived level of distraction the olfactory media caused

The curves of both graphs are relatively straight in nature, although they also both have a slightly noticeable dip at a skew of 0 s, showing that opinions were slightly more negative, as expected, when the media objects were perfectly synchronised. In addition, the mean opinions reflecting to what degree participants found the added olfactory data distracting was comparable to how annoying participants found the olfactory media. Thus, even though participants noticed the presence of the synchronisation error at some skew levels, the synchronisation errors were acceptable to the user as these results have generally shown that the presence of skew did not result in any significant negative effect. 


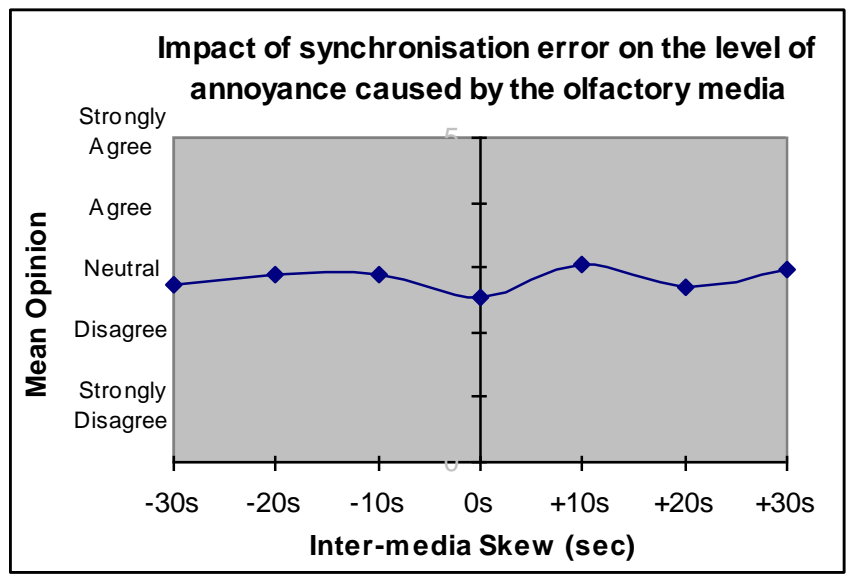

Fig. 8. Impact of inter-media skew on the perceived level of annoyance the olfactory media caused

\section{4) Impact of inter-media skew perceived level of enjoyment of the multimedia experience}

Lastly, we consider the impact of inter-media skew on the perceived level of enjoyment of the olfaction-enhanced multimedia video clips (Fig. 9). To this end, we analyse the data collated from participants' response to the questionnaire statement, 'I enjoyed watching the video clip'. An MannWhitney test showed that with regards to participants' enjoyment of the multimedia experience, there was no significant difference in their opinions when a synchronisation error was present or not.

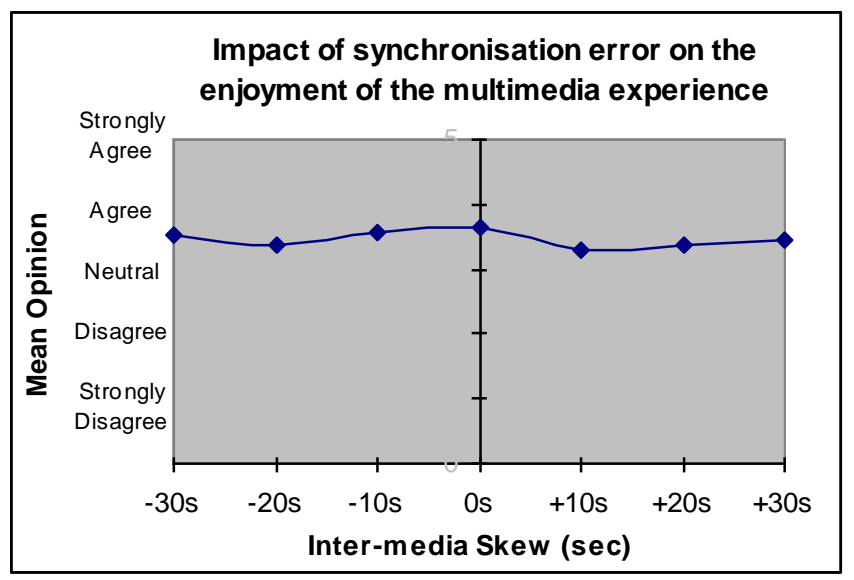

Fig. 9. Impact of inter-media skew on the perceived level of enjoyment of the olfaction-enhanced multimedia experience

\section{V.CONCLUDING DISCUSSION}

This paper has described and reported on the findings from an experimental study conducted to investigate the impact of inter-media skew variation on a users' perception of olfactionenhanced multimedia videos. Inter-media skew variation was achieved by showing participants olfaction-enhanced multimedia video clips with artificially created skews, ranging in value from -30 s to +30 s and including 0 s, between olfactory media and audiovisual media content. Accordingly, we measured the impact of the introduced skew on user satisfaction and enjoyment of the olfaction-enhanced multimedia experience.

Results showed that significant differences of participants' perception of the synchronisation effect between olfactory media and video content occurred at inter-media skew values of $-30 \mathrm{~s}$, for olfaction ahead of audiovisual, and $+20 \mathrm{~s}$ and $+30 \mathrm{~s}$, for when olfaction was behind audiovisual content. Consequently, we were able to conclude that the temporal boundaries for creating a scented audiovisual experience that will be perceived as being synchronised by users lie within a temporal range of $-30 \mathrm{~s}$ to $+20 \mathrm{~s}$. Accordingly, we propose that the inter-media skew requirements for combining video with olfaction are -30 s (olfaction ahead of video) and +20 s (video ahead of olfaction). These inter-media skew synchronisation requirements, as proposed here, are applicable to the creation of scented audiovisual experiences for users, e.g. scented videos and multi-sensory virtual reality experiences.

Furthermore, our results also revealed that although participants detected the presence of synchronisation errors, it did not have a significant impact on the general perceived quality of experience of the olfaction-enhanced multimedia for participants. Participants' opinions still reflected positive attitudes with regards to the enjoyment of the multimedia experience, the sense of relevance of the olfactory media and the sense of reality they felt it created, both in the absence and presence of inter-media skew. The slight exception to this general opinion concerns participants' perception of the sense of reality of the olfaction-enhanced multimedia. In this case, participants' opinions reflected that at a skew of $+30 \mathrm{~s}$ (olfaction behind video), the olfactory media no longer had the impact to heighten the sense of reality of the multimedia experience. In addition, participants generally did not find the delayed or advance emission of scent distracting or annoying, and their opinions mostly reflected a negative bias or neutral opinion in this respect, both in the absence or presence of inter-media skew.

Last but not least, our study raises possibilities for interesting future work, in particular as regards the influence of content on the user experience of olfaction-enhanced multimedia.

\section{REFERENCES}

[1] Berglund, B., Berglund, U. \& Lindvall, T. 1986, "Theory and methods for odor evaluation", Experientia, vol. 42, no. 3, pp. 280-287.

[2] Blakowski, G. \& Steinmetz, R. 1996, "A Media Synchronization Survey: Reference Model, Specification, and Case Studies", IEEE Journal on Selected Areas in Communications, vol. 14, no. 1, pp. 5-35.

[3] Bodnar, A., Corbett, R. \& Nekrasovski, D., 2004, "AROMA: Ambient Awareness Through Olfaction in a Messaging Application", in Proceedings of the 6th International Conference on Multimodal Interfaces, State College, PA, USA, pp. $183-190$.

[4] Brewster, S. A., McGookin, D. K. \& Miller, C. A., 2006, 'Olfoto: Designing a Smell-Based Interaction', in Proceedings of the Conference on Human Factors in Computing Systems, Montréal, Québec, Canada, pp. $653-662$

[5] Cain, W.S. \& Polak, E.H. 1992, "Olfactory adaptation as an aspect of odor similarity", Chemical Senses, vol. 17, no. 5, pp. 481-491.

[6] Chastrette, M. 2002, "Classification of Odors and Structure-Odor Relationships" in Olfaction, Taste, and Cognition, eds. C. Rouby, B. Schaal, D. Dubois, R. Gervais \& A. Holley, Paperback Re-issue edn, Cambridge University Press, New York, USA, pp. 100-116. 
[7] Chen, J.Y.C. \& Thropp, J.E., 2007, "Review of Low Frame Rate Effects on Human Performance", IEEE Transactions on Systems, Man and Cybernetics - Part A: Systems and Humans, vol. 37, no. 6, pp. 1063-1076.

[8] Dalton, P. 2000, "Psychophysical and behavioral characteristics of olfactory adaptation", Chemical Senses, vol. 25, no. 4, pp. 487-492.

[9] Drogseth, D. 2006, Quality of experience makes technology 'humanly relevant', Network World Inc., NetworkWorld.com, Available: http://www.networkworld.com/newsletters/nsm/2006/0220nsm1.html [Accessed: April 12, 2007.

[10] Gulliver, S.R. \& Ghinea, G., 2009, “A Perceptual Comparison of Empirical and Predictive Region-of-Interest Video", IEEE Transactions on Systems, Man and Cybernetics - Part A: Systems and Humans, vol. 39, no.4, pp. 744-753.

[11] Kaye, J.N. 2001, Symbolic Olfactory Display, Master of Science edn, Massachusetts Institute of Technology, Massachusetts, U.S.A. Available: http://www.media.mit.edu/ jofish/thesis/ [Accessed: September 11, 2005].

[12] Laing, D.G. 1983, "Natural sniffing gives optimum odour perception for humans", Perception, vol. 12, no. 2, pp. 99-117.

[13] Little, T. D. C. \& Ghafoor, A. 1990, "Network considerations for distributed multimedia object composition and communication", IEEE Network, vol. 4, no. 6, pp. 32-40, 45-49.

[14] Lu, G. 1996, Communication and Computing for Distributed Multimedia Systems, Artech House, Inc., Norwood, England.

[15] Rowe, L.A. \& Jain, R. 2005, "ACM SIGMM retreat report on future directions in multimedia research", ACM Transactions on Multimedia Computing, Communications, and Applications, vol. 1, no. 1, pp. 3-13.

[16] Saito, S., Ayabe-Kanamura, S., Takashima, Y., Gotow, N., Naito, N., Nozawa, T., Mise, M., Deguchi, Y. \& Kobayakawa, T., 2006, "Development of a Smell Identification Test Using a Novel Stick-Type Odor Presentation Kit", Chemical Senses, vol. 31, no. 4, pp.379-391.

[17] Steinmetz, R. \& Nahrstedt, K. 1995, Multimedia: Computing, Communications and Applications, Prentice Hall, New Jersey, USA.

[18] Steinmetz, R. 1996, "Human Perception of Jitter and Media Synchronization", IEEE Journal on Selected Areas in Communications, vol. 14, no. 1, pp. 61-72.

[19] Stephen, P. \& Hornby, S., 1997, 'Simple statistics for library and information professionals Library Association', London.
[20] Todrank, J., Wysock, C.J. \& Beauchamp, G.K. 1991, "The effects of adaptation on the perception of similar and dissimilar odors", Chemical Senses, vol. 16, no. 5, pp. 467-482.

[21] Washburn, D.A., Jones, L.M., Satya, R.V., Bowers, C.A. \& Cortes, A. 2003, Olfactory Use in Virtual Environment Training, Modelling \& Simulation, San Diego, CA. Available: http://www.modelingandsimulation.org/issue7/olfactory.html [Accessed: August 17, 2007].

[22] Zhou,Z., Cheok, A.D., Qiu,Y. \& Yang, X., 2007, "The Role of 3-D Sound in Human Reaction and Performance in Augmented Reality Environment"', IEEE Transactions on Systems, Man and Cybernetics Part A: Systems and Humans, vol. 37, no. 2, pp. 262-272.

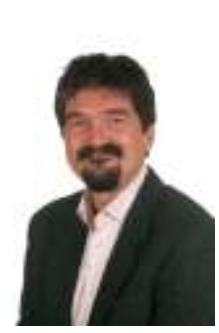

Gheorghita Ghinea (M'02) received the B.Sc. and B.Sc. (Hons.) degrees in computer science and mathematics, the M.Sc. degree in computer science from the University of the Witwatersrand, Johannesburg, South Africa, in 1993, 1994, and 1996, respectively, and the Ph.D. degree in computer science from the University of Reading, Berkshire, U.K., in 2000

He is currently a Reader with the Department of Information Systems and Computing, School of Information Systems, Computing and Mathematics, Brunel University, Uxbridge, U.K. His research interests span perpetual aspects of multimedia, quality of service, multimedia resource allocation, and computer networking and security issues.

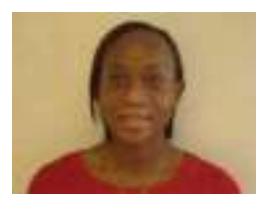

Oluwakemi A. Ademoye received a B.Sc. degree in Computer Science, in 1996, from the University of Benin, Nigeria; she then received a M.Sc. degree in Distributed Information Systems, and a Ph.D. degree in Information Systems \& Computing, from Brunel University, United Kingdom, in 2003 and 2008 respectively. Her research interests focus on multimedia and olfaction. 